\title{
ChickTrack - A Quantitative Tracking Tool for Measuring Chicken Activity
}

This paper was downloaded from TechRxiv (https://www.techrxiv.org).

LICENSE

CC BY-NC-SA 4.0

SUBMISSION DATE / POSTED DATE

$21-07-2021 / 26-07-2021$

CITATION

Neethirajan, Suresh (2021): ChickTrack - A Quantitative Tracking Tool for Measuring Chicken Activity. TechRxiv. Preprint. https://doi.org/10.36227/techrxiv.15031440.v1

DOI

10.36227/techrxiv.15031440.v1 


\title{
ChickTrack - A Quantitative Tracking Tool for Measuring Chicken Activity
}

\author{
Suresh Neethirajan ${ }^{1 *}$ \\ ${ }^{1}$ Farmworx, Animal Sciences Department, Wageningen University, The Netherlands \\ *Correspondence: suresh.neethirajan@wur.nl
}

\begin{abstract}
The automatic detection, counting and tracking of individual and flocked chickens in the poultry industry is of paramount to enhance farming productivity and animal welfare. Due to methodological difficulties, such as the complex background of images, varying lighting conditions, and occlusions from e.g., feeding stations, water nipple stations and barriers in the chicken rearing production floor, it is a challenging task to automatically recognize and track birds using computer software. Here, a deep learning model based on You Only Look Once (Yolov5) is proposed for detecting domesticated chickens from videos with varying complex backgrounds. A multiscale feature is being adapted to the Yolov5 network for mapping modules in the counting and tracking of the trajectories of the chickens. The Yolov5 network was trained and tested on our dataset which resulted in an enhanced tracking precision accuracy. Using Kalman Filter, the proposed model was able to track multiple chickens simultaneously with the focus to associate individual chickens across the frames of the video for real time and online applications. By being able to detect the chickens amid diverse background interference and counting them precisely along with tracking the movement and measuring their travelled path and direction, the proposed model provides excellent performance for on-farm applications. Artificial intelligence enabled automatic measurements of chicken behavior on-farm using cameras offers continuous monitoring of the chicken's ability to perch, walk, interact with other birds and the farm environment, as well as the assessment of dustbathing, thigmotaxis, and foraging frequency, which are important indicators for their ability to express natural behaviors. This study highlights the potential of automated monitoring of poultry through the usage of ChickTrack model as a digital tool in enabling science-based animal husbandry practices and thereby promote positive welfare for chickens in animal farming.
\end{abstract}

Keywords: Precision livestock farming; digital agriculture; Yolo; tracking; deep learning; chicken automated measurements; multi-target detection and tracking.

\section{Introduction}

The world's growing population is dependent on animal agriculture. Animal products provide nutritious meals that help feed and sustain communities globally. Recent data shows that the global poultry market is expected to grow by $\$ 422.97$ billion by the year 2025 at a growth rate of $7 \%$ per annum [1]. Although debatable, it is perceived that poultry farming contributes less to climate change in comparison to cattle farming because of less methane emissions, relatively less resources needed, and higher feed conversion ratio [2]. As the global demand for animal products continues to grow, the agricultural industry must continue to advance its quality and efficiency of production [3], while simultaneously ensuring good poultry health and welfare. Good animal welfare requires not only good physical health, but also mental health through minimizing suffering and promoting 
positive experiences for the animals [4]. However, such traits are challenging to objectively, efficiently, and timely record in a farm containing over thousands of individual animals.

To support the increasing agricultural demand and ensure biosecurity adherence and operational efficiency for the animals, farm video surveillance systems are expected to grow to US $\$ 3.6$ billion by 2027 [5]. Remote monitoring, physiological and behavioral phenotyping data collection through sensors, vast data storage and rapid data transfer have advanced precision livestock farming in the last 20 years $[6,7,8]$. However, the integration of smart sensing technologies, including the use of videos within the intelligent livestock surveillance systems, have to overcome technical challenges for large-scale phenotyping to be possible. The incorporation of Artificial Intelligence (AI) offers less stressful management options of poultry. Due to the ability of AI to monitor large number of welfare indicating parameters; continuous data collection and processing and real-time instant decision-making features; the industries are considering evaluation of AI based tools in the poultry value chain.

\section{Automatic Monitoring Surveillance}

To support the growing agricultural industry, automated measurement systems are emerging as useful tools to monitor and promote good animal welfare. There are currently significant animal welfare challenges facing the agricultural industry, especially the poultry industry. Current poultry farming practices result in the chicken's death before slaughter and rejection of billions of male chicks that are immediately killed after hatching in the egg industry, which is just the system's design as they are "useless" regarding egg production, on an annual basis before they are processed for meat [3]. In the poultry meat industry, often chickens are rejected at the slaughterhouses due to the lack of sufficient meat quality and bruises, skin injuries, fractures, or other lesions on the chicken bodies. This loss of life is of a significant concern for animal welfare, agricultural efficiency, and economic impacts [3]. The positive, negative and neutral chicken welfare indicators based on video and image analysis can be derived from early-life stress due to separation of the mother-chick, very high density, bad air circulation, poor hygiene leading to respiratory issues, injuries on their feet due to ammonia building up on the ground, bad housing environment, no positive / rewarding stimuli (playful behavior), behavioral problems such as pecking or cannibalism, chronic stress, peak in stress before slaughter, suffering when the slaughtering method is not as efficient, unnatural lighting conditions and others. The link between poultry health and the poultry product quality emanates from the human risk of diseases if the animals have been infected before, [9] and the influence of stress hormones on meat qualities [10]. Few studies have demonstrated cameras integrated with instrumentation systems of Artificial Intelligence (AI) can assess flocks for health concerns, thus improving the survival rate and product quality of farmed poultry [3].

To improve the welfare of farm animals, the needs of both the individual animals and the needs of the group (herd and flock) must be considered. Advancements in sensors and instrumentation technology allow the capture of behavioral, physiological, and productivity measurements of individual animals $[11,12]$. The automated tracking systems can detect and predict behaviors that harm animals such as cannibalism and feather pecking; measuring feed consumption; enhancing production and welfare; light-based movement activity; and quantifying in separate areas to understand preferences of the birds within the pens $[13,14]$. Due to the surge in the sensor-enabled 
technologies, now it is feasible to collect video and other physiological data more often consistently on an individual animal basis [15]. This is important because not all farm animal species can be measured the same way. Because of the size, shape and the relatively longer life period of cattle and pigs, individual monitoring is easier compared to poultry. Hence, with the aid of Artificial Intelligence, automated monitoring tools can offer the same quality of measurement for individual poultry birds.

Different methods to monitor individual animal behavior exist, ranging from inserted chips that continuously recorded physiological measures, to wearable sensors and (thermal) imaging. Each method has its advantages and disadvantages and can be employed depending on the purpose of monitoring. Some researchers $[16,17,18]$ have used wearable sensors attached to the birds' legs to measure the activity and movement of birds, but for commercial settings, this is not feasible due to the technological limitations and high costs. Hence, optical flow-based video assessments would be ideal for monitoring of poultry behavior and physiology. Another form of automated tracking comes from video-based tracking.

Video based tracking is superior to animal wearable sensors in terms of measuring biometric features such as activity, movement and predicting diseases. This is because of the practical scalability of the measurements. And, by eliminating the capturing and handling of the birds, this minimizes distress.

To address the growing sector of precision livestock farming, the development of automatic monitoring and surveillance systems for animal behavior and phenotyping, as well as real-time assessments using video and image analysis are gaining momentum. Temperature of the chicken body parts from thermal imaging [19], inter-individual interactions of chicken, movement [20], automated weighing of the animals to keep track of productivity [21], machine vision-based eggcounting systems are some ways by which phenotyping data can be automatically measured using monitoring systems. Automated monitoring, surveillance, and assessments are of immediate need for the poultry indicators such as individual tracking; genome wide association investigations for optimization of breeding; maintaining the individual animal identity; tracking the activity and space usage continuously; group level activity assessment; to be able to differentiate between individual animals; early detection of deviating patterns; social and behavioral problem detection in chickens; comparing the activities of current flock with past flocks; detect and count laying hens; analyzing the preference of light intensity of individuals and groups of hens and poultry birds; range use and fearfulness in free-range hens; keel bone fracture assessment from activity using video monitoring.

\section{Challenges}

Different aspects of today's livestock production have been shown to be stressful and challenging for animals, from early-life stages to the moments before slaughter. Larger flocks result in lower margins for farmers. As the industry strives to use fewer inputs for more sustainable production, it is inevitable that solutions for preventing disease have to be found, which would result in enhanced disease detection and positive poultry welfare. 
In the poultry sector, machine vision focused research has developed tools in behavioral detection based on the quantification of the brightness patterns within a two-dimensional video [22]. However, there is a need for models and tools that allow multiple chickens to be detected and monitored.

The best way to prevent the missing animals or the poultry bodily features due to occlusions and related losses in the visual based measurement is through the individualistic and/or group level evaluation of animals on a continuous basis [3]. On large-scale commercial farms, such attention to detail has been considered to be inaccurate and inefficient, but with the integration of Artificial Intelligence (AI) assisted technology, individualized and per-herd assessments of livestock are possible and accurate [3].

Farmers need autonomous tools to be able to obtain insights on their animals that might reveal indication of their welfare. To promote good animal welfare, farmers need assistance with the automated surveillance of the animals. By continuously monitoring animals, farmers are able to reliably detect the animal's needs. Telltale signs from visual video analysis alone can provide practical information on a daily basis by scoring injuries, lameness, feeding events, and measuring the animals' behavioral records of activity, social interactions, and emotions. The precision of the system allows for animals to be continuously monitored. This monitoring helps farmers in return to improve the health and welfare of their animals and become more competitive within their industry. For real-time practical applications, it is necessary to design a model to ensure not only the accuracy of the detection but also to satisfy the complexity associated to lighting conditions and multiplexing additional features such as counting and tracking.

\section{Related Works}


Table 1: Research on Chicken (Poultry) detection based on deep learning technology.

\begin{tabular}{|c|c|c|c|c|c|}
\hline Networks model (Algorithms) & Applications & $\begin{array}{l}\text { Dataset } \\
\text { volume }\end{array}$ & $\begin{array}{c}\text { Data collection } \\
\text { location }\end{array}$ & $\begin{array}{c}\text { Measurement } \\
\text { accuracy }\end{array}$ & References \\
\hline $\begin{array}{c}\text { Chicken sound convolutional } \\
\text { neural network (No videos or } \\
\text { images data) }\end{array}$ & $\begin{array}{c}\text { Avian influenza } \\
\text { detection }\end{array}$ & $\begin{array}{l}\text { Audio files } \\
\text { from } 5 \\
\text { chickens }\end{array}$ & $\begin{array}{l}\text { Controlled } \\
\text { biosafety } \\
\text { laboratory }\end{array}$ & $97.4 \%$ & {$[23]$} \\
\hline Convolutional neural network & Behavior assessment & $\begin{array}{l}12000 \text { images } \\
\text { from } 3087 \\
\text { chickens }\end{array}$ & Chicken Coop & $99.17 \%$ & {$[24]$} \\
\hline $\begin{array}{c}\text { You Only Look Once }+ \\
\text { Multilayer Residual Module } \\
\text { (Yolo + MRM) }\end{array}$ & $\begin{array}{l}\text { Stunned state of } \\
\text { broilers }\end{array}$ & $\begin{array}{l}2319 \text { images } \\
\text { from } 12 \text { broiler } \\
\text { chickens }\end{array}$ & $\begin{array}{l}\text { Animal care } \\
\text { facility }\end{array}$ & $94.74 \%$ & {$[25]$} \\
\hline $\begin{array}{c}\text { Deeplabcut and pretrained } \\
\text { ResNet-50 }\end{array}$ & $\begin{array}{l}\text { Chicken pose } \\
\text { estimation \& behavior } \\
\text { classification }\end{array}$ & $\begin{array}{l}28 \text { videos from } \\
\text { only } 4 \text { broiler } \\
\text { chickens }\end{array}$ & $\begin{array}{l}\text { Controlled } \\
\text { laboratory } \\
\text { facility }\end{array}$ & $\begin{array}{c}0.7511 \\
\text { (standing), } \\
0.5135 \\
\text { (walking), } \\
0.6270 \\
\text { (running), } \\
\text { 0.9361 (eating) } \\
\text { accuracies }\end{array}$ & {$[26]$} \\
\hline VGGNet-16 and ResNet-50 & $\begin{array}{l}\text { Chicken disease } \\
\text { identification }\end{array}$ & $\begin{array}{l}600 \text { images } \\
\text { from } 5 \\
\text { chickens }\end{array}$ & $\begin{array}{l}\text { Laboratory } \\
\text { facility }\end{array}$ & $\begin{array}{c}66.91 \% \\
\text { accuracy }\end{array}$ & {$[27]$} \\
\hline $\begin{array}{c}\text { Single Shot MultiBox Detector } \\
\text { (SSD) model, with InceptionV3 } \\
\text { as the backbone }\end{array}$ & $\begin{array}{c}\text { Chicken disease } \\
\text { detection }\end{array}$ & $\begin{array}{l}6601 \text { photos of } \\
\text { white broilers } \\
\text { and } \\
4296 \text { photos of } \\
\text { jute broilers }\end{array}$ & $\begin{array}{l}\text { Commercial } \\
\text { poultry house } \\
\text { and outdoors }\end{array}$ & $\begin{array}{l}99.7 \% \text { mean } \\
\text { average } \\
\text { precision }\end{array}$ & {$[28]$} \\
\hline Convolutional neural networks & $\begin{array}{l}\text { Monitoring heat stress } \\
\text { of chickens }\end{array}$ & $\begin{array}{l}25,000 \text { images } \\
\text { from } 10 \text { broiler } \\
\text { chickens }\end{array}$ & $\begin{array}{l}\text { Controlled } \\
\text { environment } \\
\text { chicken } \\
\text { coop/cage (240 }\end{array}$ & $95 \%$ & [29] \\
\hline
\end{tabular}




\begin{tabular}{|c|c|c|c|c|c|}
\hline $\begin{array}{l}\text { Support Vector Machine (SVM- } \\
\text { Machine Learning model) }\end{array}$ & $\begin{array}{c}\text { Detection and } \\
\text { prediction of broiler } \\
\text { chicken }\end{array}$ & $\begin{array}{l}23,996 \text { images } \\
\text { from } 2 \text { groups } \\
\text { of } 20 \text { broilers }\end{array}$ & $\begin{array}{l}\mathrm{cm} \times 240 \mathrm{~cm} \times \\
210 \mathrm{~m}) \\
\text { Isolated controlled } \\
\text { environment } \\
\text { chambers }\end{array}$ & $97.8 \%$ & {$[30]$} \\
\hline $\begin{array}{l}\text { Fully Convolutional Networks } \\
\qquad(\mathrm{FCN})\end{array}$ & $\begin{array}{c}\text { Density map } \\
\text { estimation }\end{array}$ & $\begin{array}{l}\text { A total of } 100 \\
\text { images }\end{array}$ & Chicken coop & $16 \%$ & [31] \\
\hline Yolov3 & $\begin{array}{l}\text { Behavior of laying } \\
\text { hens }\end{array}$ & $\begin{array}{l}\text { 10,230 images } \\
\text { from } 18 \text { laying } \\
\text { hens }\end{array}$ & $\begin{array}{l}\text { Wire cage with two } \\
\text { pens, } \\
\text { each of } 120 \mathrm{~cm} \mathrm{X} \\
120 \mathrm{~cm} \mathrm{X} 70 \mathrm{~cm}\end{array}$ & $\begin{array}{c}\text { mate } \\
(94.72 \%), \\
\text { stand } \\
(94.57 \%), \\
\text { feed } \\
(93.10 \%), \\
\text { spread } \\
(92.02 \%), \\
\text { fight } \\
(88.67 \%) \\
\text { and drink } \\
(86.88 \%)\end{array}$ & {$[32]$} \\
\hline
\end{tabular}


Studies use deep learning technologies for applications such as disease detection or behavioral classification in avian species have only recently been growing (Table 1). However, no research has been published yet on the detection, counting and tracking of the chickens under occlusion conditions nor using the You Only Look Once software (Yolov5). Tracking of chicken movement is achieved through taking an initial set of the chicken shape and contour detections, creating a unique ID based on the coordinates in the image (frames from videos) for each of the initial detections, and then tracking as they move around frames in the video, continuing the ID assigned. Occlusion, background clutter and change in appearance are some of the challenges in the detection and tracking of chicken movement. Occlusion occurs when the chicken gets hidden by another object such as a feeder or another chicken. Between the frames of the video, there is a higher possibility that the chicken may disappear and reappear again. Feeders, water nipple providers or other objects in the chicken rearing floor may have similar colors or textures to the chicken feathers and it may become harder to track results with the cluttered background. Different viewpoints of the chicken based on the camera positioning and camera angle may capture videos that may acquire the chicken's look very differently and without a context, this might lead to difficulties identifying the chicken.

Furthermore, no research work has been reported to date on the recognition, detection, counting, tracking, and measuring the trajectory motion path of the activity of chickens. The datasets of the studied mentioned in Table 1 were relatively small, and although the accuracy was shown to be high, the performance accuracy would be insufficient in real-time outside of controlled conditions. Moreover, the integration of various activities by one model is required for complex movementbased data collection concerning the chicken activity and detection.

Therefore, a Deepsort Yolov5 based model was developed in the present study to ensure the accuracy of the detection as well as the requirements for real-time motion and activity monitoring using the domesticated chicken (Gallus gallus domesticus). Yolov5 is a relatively recently developed software created by Ultralytics in 2020 [33] and offers superior detection accuracy and real-time performance. The proposed model provides support for accurate, real-time detection of chicken activity individually and on the flock level. Locomotion, speed of walking, distance travelled or walked by the chicken, feeding interval based on the movement and related behavioral characterizations as well as where the chickens are located in the pen can be measured as part of the chicken activity.

Identification and tracking of identical looking unmarked birds in large flocks is tough, demanding and time-consuming, but there is an immediate and definitive need for such automated measurement systems in farm animals including poultry. The overall goal of this study was to develop a model based on machine learning algorithms that will convert heterogeneous data that are collected via automated video recording systems for measuring the phenotypes of chickens. There is no classification involved in this study, as the goal is to detect the chicken, count and track the path of movement of the chicken. 


\section{Materials and Methods}

\section{Dataset characteristics}

Our dataset is composed of a total of 72 chicken (White Leghorn breed, Plymouth Rock, Rhode Island Reds) videos were acquired during different times of the day and in varying background lighting conditions (Figure 1) recorded in two poultry farms in Ontario, Canada. RGB cameras were deployed at varying heights and in varying lighting conditions inside the pens outside the coop, and outside in the free roaming zone. The videos were recorded with a resolution of $1280 \mathrm{X}$ 720 pixels (frame width $\mathrm{X}$ height) at 30 frames per second. The total length of all the videos for all the breeds together was over 8 hours. Frames were annotated using the opensource graphical annotation LabelImg software [34] and the contours were labeled by bounding box. Annotation classes of chickens were used and all the chickens in the frame were annotated.

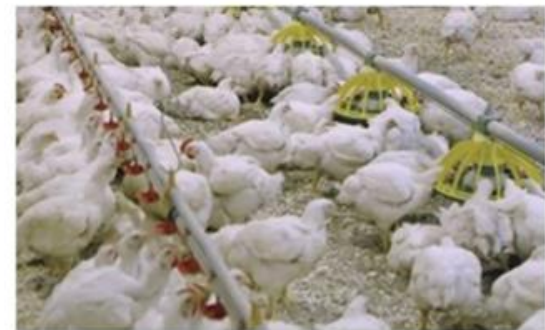

(a)

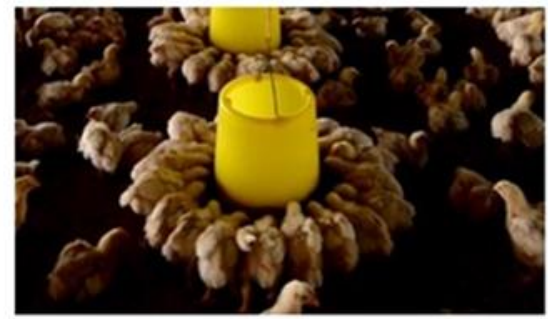

(d)

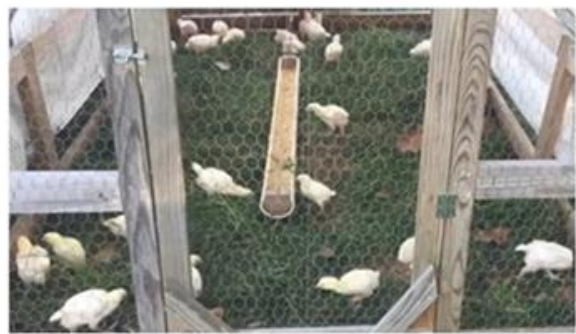

(b)

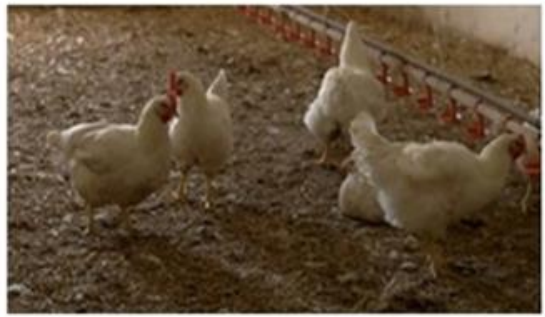

(e)

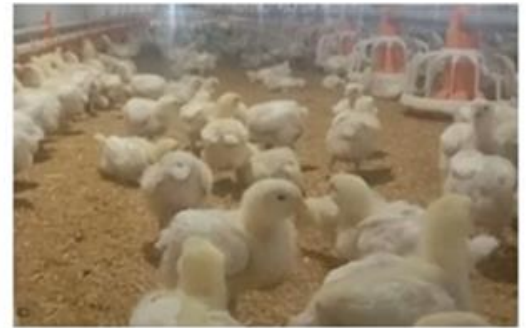

(c)

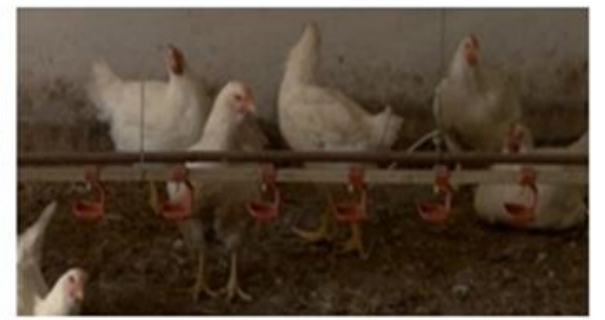

(f)

Figure 1: Typical examples of images from the video dataset of chickens obtained in the free range, commercial and open poultry farms. Chickens are housed in varying background conditions. (a) Chickens occluded by feeders and watering nipples (b) Free range chickens occluded by wire mesh barrier (c) Front light angle (d) Poor lighting condition (e) Sidelight angle (f) Backlight angle.

\section{ChickTrack Detector Model and Architecture}

To take advantage of the advancement of Convolutional Neural Networks (CNN) based detection, in this project Yolov5 and DeepSort were utilized. Yolo is a single stage detection technique without a distinct region proposal and treats the detection of the target as a single regression problem [35]. Object detection using Yolov5 has been demonstrated as a superior way in comparison to other target detection and recognition algorithms [36,37,38,39]. Yolov5 has the advantages of rapid processing time in the deep learning network; ability to handle larger datasets 
and real-time continuous detection [40,41]. The proposed Yolov5 based ChickTrack detector model exploits the class probabilities in recognition of the chickens and the bounding box coordinates for enabling the measurement of trajectory paths between the frames of the video data. Figure 2 shows the schematic of the proposed ChickTrack Yolov5 detector model showing the backbone architecture and the last layers of the detector. For the training of the model and testing, a high-performance computer workstation was used, and the details concerning GPU and configurations are shown in Table 2.

The proposed Yolov5 framework consists of 3 major multi-scale modules: feature extraction and thereby detection of chickens; counting of chickens; and the tracking motion path. The frames from videos were inputted into the Yolov5 model. By inputting videos into the frame network architecture, Yolov5 creates layers and extracts features such as the boundary of the chicken and the centroid of chicken body, followed by feature mapping the output object. The detector module of the ChickTrack uses the Yolov5 architecture in layering the deep neural network and produces detection at different scales kernels. This is then followed by the counting and tracking modules.

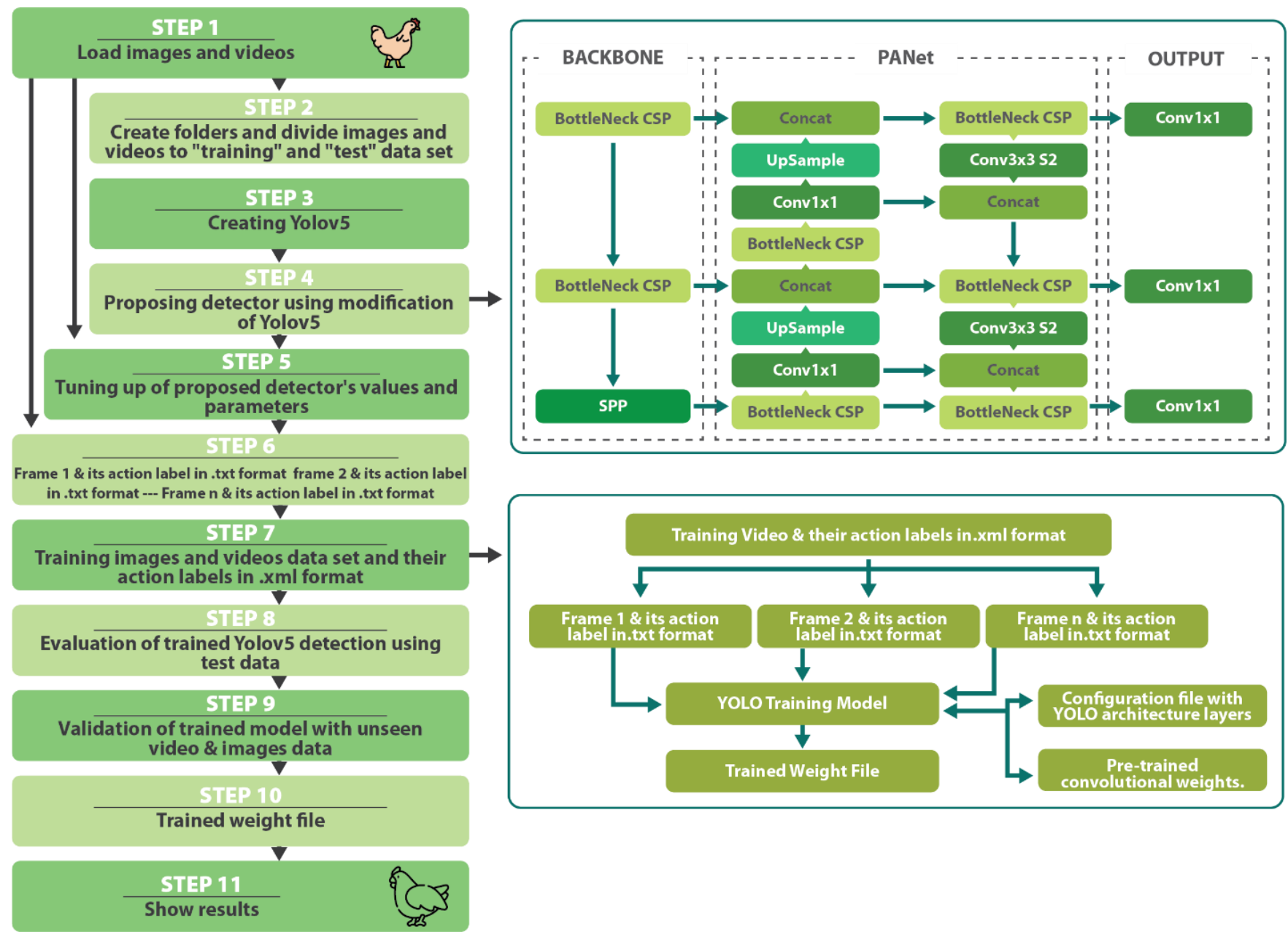

Figure 2: Architecture of Yolov5 used in the development of ChickTrack model for multi-object detection, counting and tracking. 
Table 2: The configuration of the workstation used in this study

\begin{tabular}{cc}
\hline CPU & Intel(R) Core(TM) i7-6700 CPU \\
\hline CPU basic frequency & $3.4 \mathrm{GHz}$ \\
Core/thread number & Four core / eight threads \\
Memory capacity & $24 \mathrm{~GB}$ \\
Hard drive capacity & $1 \mathrm{~TB}$ \\
Graphics card chip & Nvidia-1080 ti, 11 GB \\
Cuda & Cuda 10.1 with Cudnn 7.5.1 \\
Data Processing & Python 3.9.5, OpenCV \\
Deep Learning Framework & Pytorch 1.9.0 \\
Architecture & CSP backbone and PA-NET neck
\end{tabular}

264

\section{Experimental Analysis}

The training dataset was made of over 3800 annotated frames, where $80 \%$ of the frames were used for training, $10 \%$ for testing and $10 \%$ for validation. Towards the detection of the multiple chickens from the images, the model was trained using the dataset by adjusting the number of epochs. Training the model for 100 epochs took about $75 \mathrm{~min}$. The performance metrics for the training and validation dataset is shown in Figure 3.
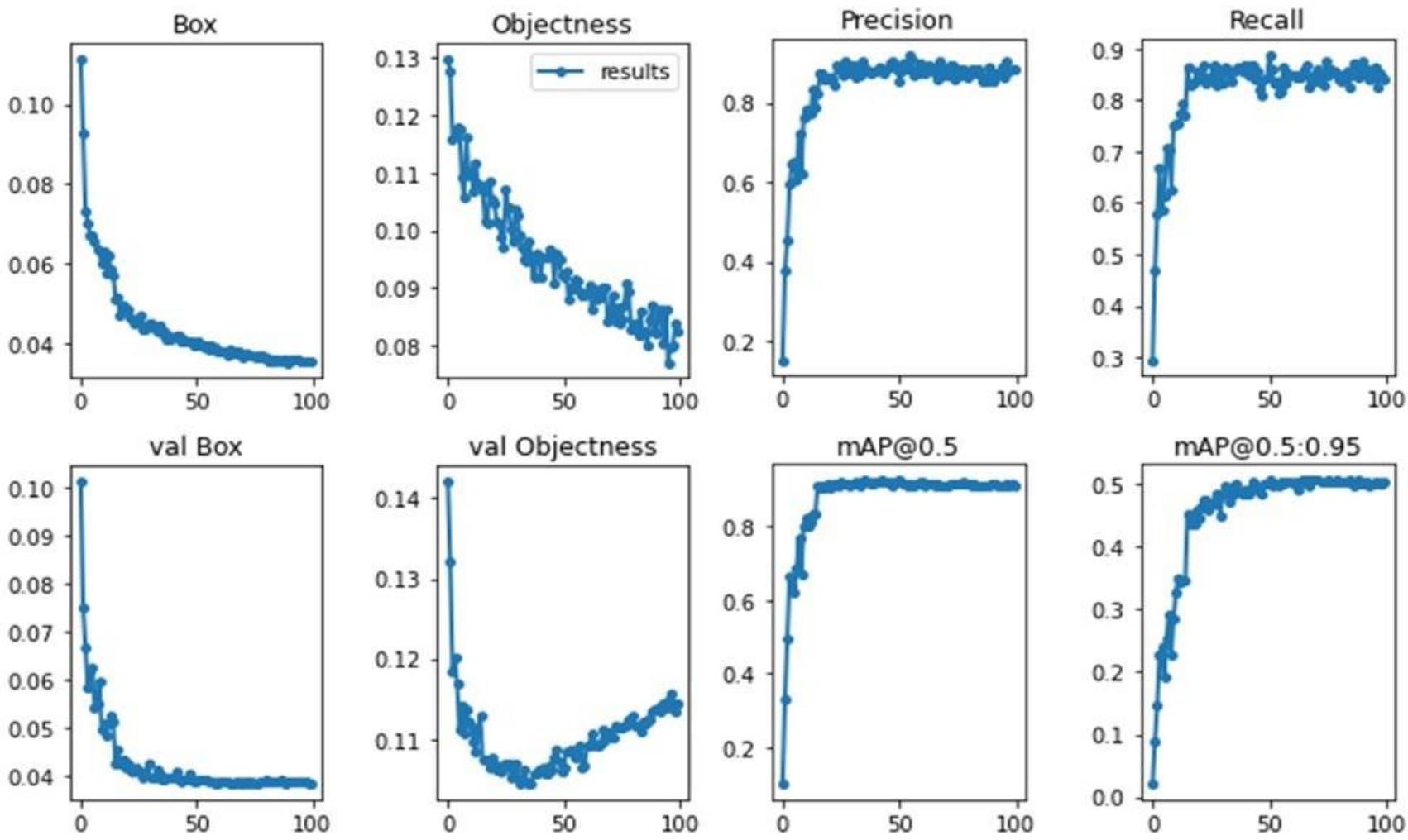

Figure 3: Selected examples of detection results based on the proposed YoloV5 approach on the chicken video dataset. Detections are labeled with red rectangle bounding box and denoted with the associated confidence scores. 
Box loss indicates how well the developed model can locate the individual chicken in the video frame and how well the predicted bounding box covers the chicken. Objectness is the measure of the probability that the chicken exists in the proposed region of interest in the video frame. The higher the objectness, the more likely the image window contains the chicken in the process of detection. Precision, mean precision and recall indices (Figure 3) show that the model improved before plateauing after around 25 epochs. Similarly, the objectness and box losses for validation data showed decline until around 25 to 30 epochs, indicating the early stopping for selection of the best weights in the training of the model.

\section{Results and Discussion}

Detecting and tracking poultry using optical flow and video based automatic assessment is a challenging task of which the outcome is to create a meaningful insight for intervention or decision-making processes for farmers. Real-time detection of activity of broilers or laying hens in poultry farms is tricky as the detection in the video involves verification of the presence of the chicken in the image sequences from the video data and precisely locating it for individual recognition and estimating of its coordinates. The tracking of the chicken's temporal and spatial changes in the video sequence includes monitoring its presence, shape or the contour surrounding the chicken body. By matching the target region of the chicken in the successive frames of sequence of images, at closely spaced time intervals, the recognition and detection can be achieved.

Optical flow has been demonstrated as a way to identify vehicles for driver assistance systems [42]; collision avoidance for multicopter Unmanned Aerial Vehicles [43]. In this study, we used optical flow as a means for establishing a framework in the detection, counting and the measurement of the movement trajectory of individual chickens. The results obtained based on the training of the proposed ChickTrack model is shown in Figure 5. In order to count individual chickens in the video frames, it is necessary to determine the relationship between the trajectory of the chicken and the counting line in the frame. In the proposed module, the direction of each chicken was calculated when the trajectory of the chicken and the counting line intersected. The chicken detection module involved the presence, the specific coordinates or the location of the chicken using the bounding box. The number of chickens in the video are also calculated by using the count of the bounding boxes. The training and validation loss curves based on the ChickTrack model performance is shown in Figure 5. The graph indicates that the loss value decreased after rapidly after 30 epochs of training and then stabilizes up to 70 epochs and further rapidly decreases after 70 epochs. Hence, the ChickTrack model output after 70 epochs was chosen as the target detection and recognition for chickens. The results from mean average precision and validation confirmed that the ChickTrack model was trained well without overfitting. 


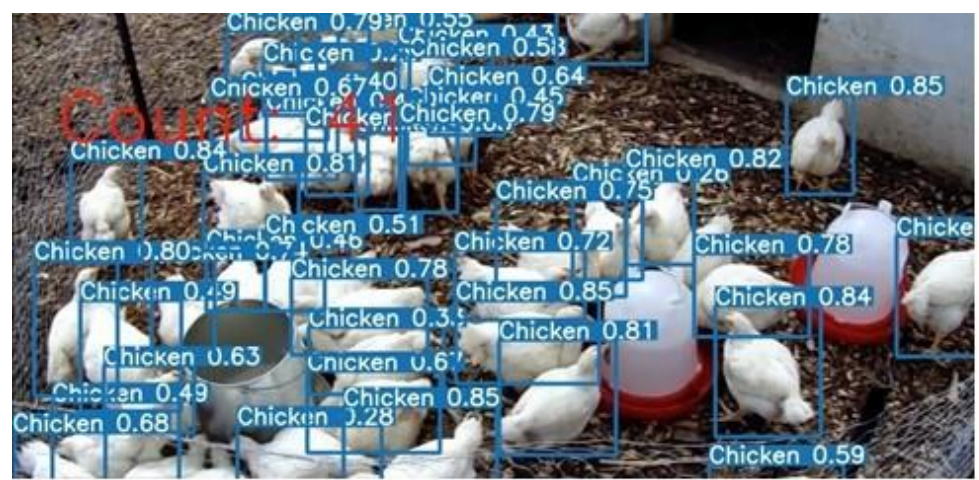

(a)

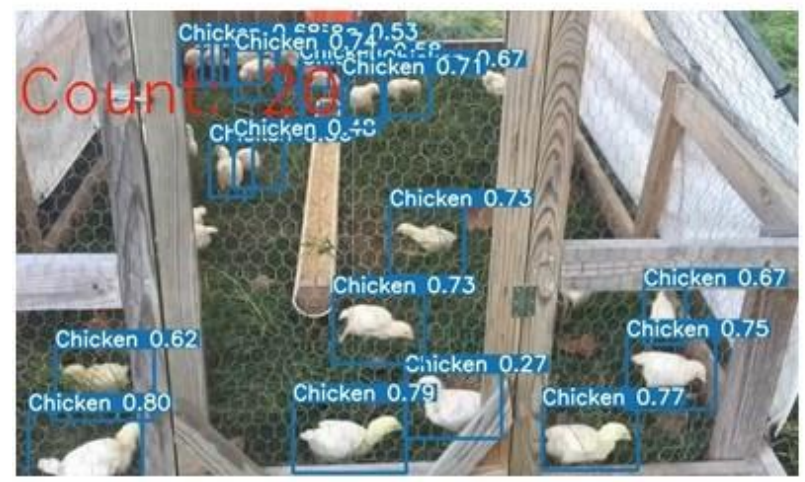

(c)

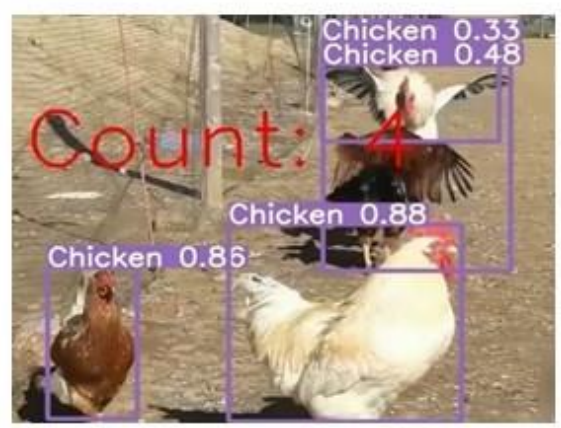

(b)

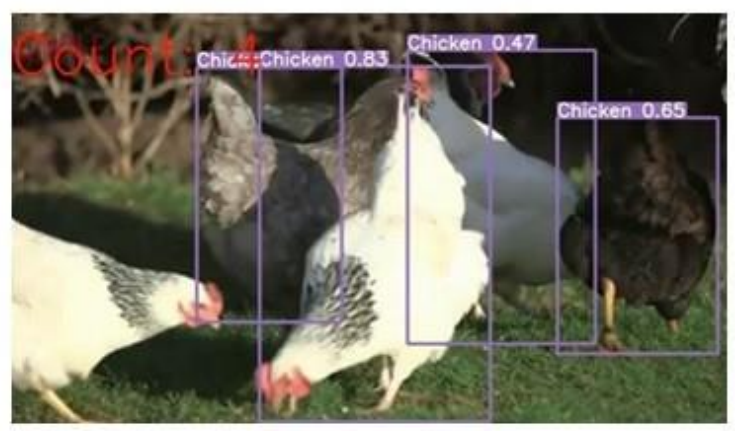

(d)

Figure 4: Sample results showing the detection and counting of chickens from the video data based on the proposed ChickTrack model.

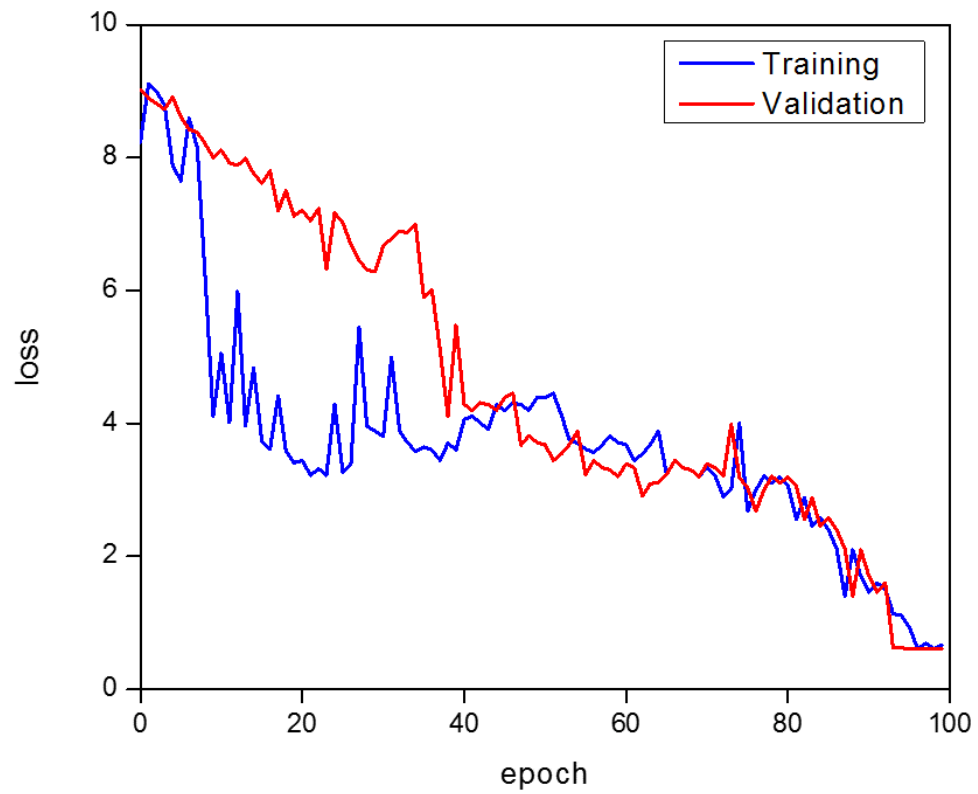

Figure 5. ChickTrack model network training results showing the training and validation losses from the poultry video datasets. 


\section{Chicken tracking}

Chicken tracking from the videos involves the process of measurement (Figure 6) of the coordinates across multiple series of frames. In the proposed model, all possible detections of the chicken in the frame were chosen and was given a centroid based ID based on the bounding box using Kalman filer. This was carried out by calculating centroids for each of the bounding boxes in the frame 1. In the subsequent frames, the same ID of that chicken was carried forward. As the frame changes, if a new chicken appears, then the old ID is dropped, and it is assigned a new ID. Hence, tracking the chicken becomes challenging due to the fact that the bird in the video may appear or disappear between the frames or there may be occlusions hiding the bird in later frames. By frame-to-frame centroid assessment, the distance from previous centroid being calculated, this challenge was overcome.

The Kalman filter models the future position and the velocity using gaussians. By using probability, the Kalman filer assigns the measurement to its prediction and updates itself. The developed model performs the tracking not just based on the distance, but also by computing deep features (both appearance cues and geometry of chickens) for each individual bounding box and uses the similarity between the deep features by factoring into the tracking logic. The dim vector for each bounding box is extracted by the model from the images of chickens in each frame of the video and acquires the key features. Hence, the model is capable of overcoming occlusions.

It should be emphasized that the efficiency of tracking and reduction of false positives is directly related to the quality of the detection module of the chickens. Hence, the performance of the tracking algorithm needs careful optimization through extensive training of the dataset. The false negative recognition and detection of chicken can be compensated through integrating visual tracking into the intersection over union. The quality of the chicken tracking module can be enhanced by reduction in the number of switches in the ID between the frames of the video data.

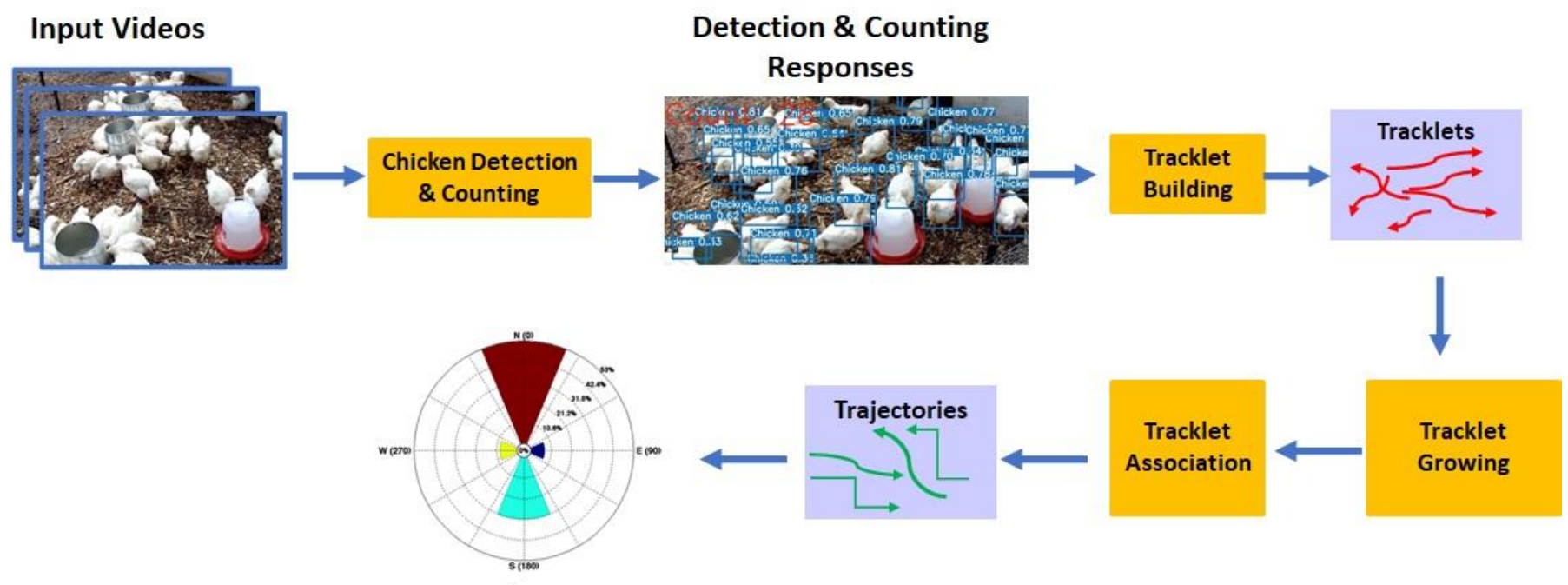

Figure 6. Illustration of the ChickTrack model's tracking framework. Along with the input video module, a real-time tracking framework is established using Kalman filter as part of the ChickTrack model to learn the responses of chicken detection in representing the features of 
tracklets and pre-processing the growth of tracklet in the video frames, and the output trajectory is

358 being established as the result.

369

Table 3 shows the overlap success rate at threshold of 0.5 for three videos analyzed (Supplementary files Video S4, Video S5, Video S6) of the proposed ChickTrack model. The trends clearly indicate that the developed model is accurate in tracking the movement of chicken between individual frames of the video. Verification results of the object trajectory is based on the direction of the chicken's movement, and the graphs (Figure 7) show the accumulative direction output showing the proportion of the movement of overall chickens in the three videos.

Table 3 Performance of the proposed chicken activity tracking framework with the Yolov5 detector

\begin{tabular}{ccccc}
\hline Data & Time & Ground Truth & Highest tracking id & Difference \\
\hline Video S4 & $0 \min 10 \mathrm{sec}$ & 16 & 18 & +2 \\
Chicken Coop & $0 \min 20 \mathrm{sec}$ & 17 & 24 & +7 \\
& $0 \min 30 \mathrm{sec}$ & 19 & 24 & +5 \\
& $0 \min 38 \mathrm{sec}$ & 28 & 23 & -5 \\
Video S5 & $0 \min 10 \mathrm{sec}$ & 48 & 42 & -6 \\
Commercial & $0 \min 40 \mathrm{sec}$ & 41 & 43 & +2 \\
Poultry Farm & $1 \mathrm{~min} 20 \mathrm{sec}$ & 48 & 34 & -4 \\
& $2 \min 0 \mathrm{sec}$ & 42 & 43 & -4 \\
& $2 \min 40 \mathrm{sec}$ & 35 & 7 & +8 \\
Video S6 Free & $0 \min 10 \mathrm{sec}$ & 5 & 3 & +2 \\
Range Farm & $0 \min 20 \mathrm{sec}$ & 4 & 7 & -1 \\
Chicken & $0 \mathrm{~min} 30 \mathrm{sec}$ & 4 & 7 & +3 \\
& $0 \mathrm{~min} 40 \mathrm{sec}$ & 3 & 323 & +3 \\
\hline Total Accuracy & & 310 & & +13 \\
\hline
\end{tabular}
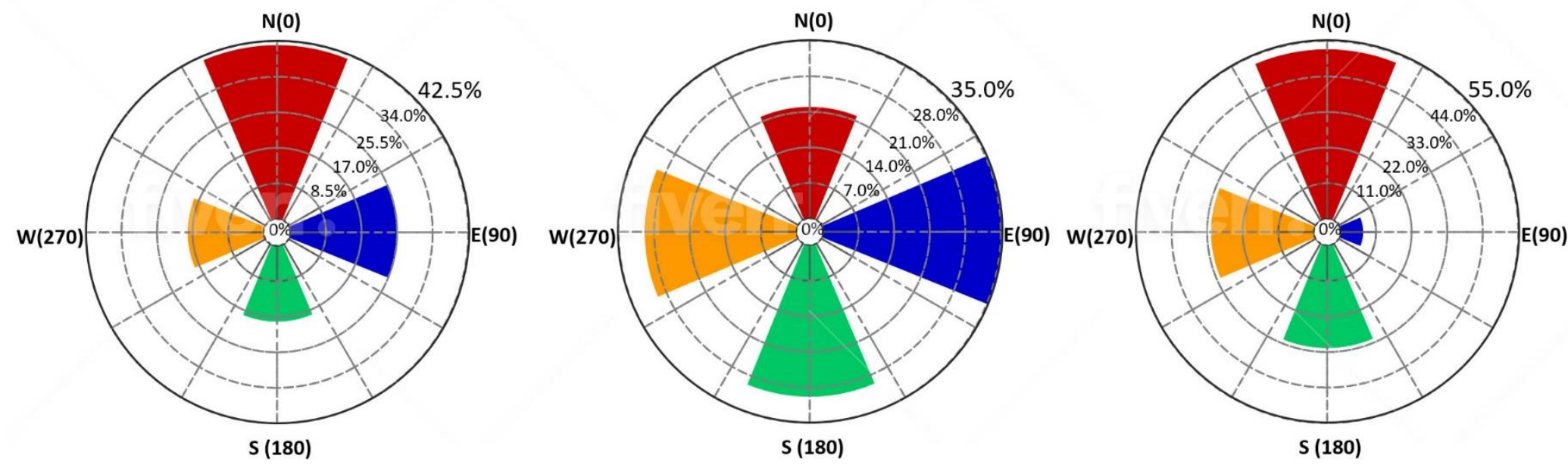

385 Figure 7. Chicken migration evaluation by 2D video imaging and tracking analysis by the 386 proposed ChickTrack model. Representative wind-rose plots show the distribution (Red - North, 387 Blue - East, Green - South, Orange - West) of the trajectory of chickens in the presence and 
absence of feeder and water nipping stations in (a) the chicken coop, (b) the commercial poultry farm and (c) the free-range open field farm.

The difference in the magnitude of each direction as chosen by the chicken population can be seen as percentages in the graphs. Preferential directional persistence of chickens as measured by the ChickTrack module shows the ability of the proposed model to assess the migration pattern. Individual chicken's net displacement analysis shows that the birds migrated more often in the northern direction for the chickens observed in the coop in comparison to the chickens in the commercial poultry farm for the analyzed dataset. By understanding the individual chicken's movement characteristics, the farmers could be able to assess the space allowance for the birds in the flooring area.

\section{Future studies}

The deep learning-based technique developed for chicken detection, counting and tracking was based on 2D video data obtained from a single camera. Currently, studies are underway to explore the enhancement of the YoloV5 model using 3D data and Kinect depth sensors from multiple cameras of the same chickens obtained from varying angles. In this study, the most common breeds namely White Leghorn chickens, Plymouth Rock, and Rhode Island Reds were used for experiments and data analysis. Additional studies with other chicken breeds would further strengthen the validation of the developed ChickTrack automated chicken movement platform. Social interactions or the social network analysis of chickens is an underexplored research area, and the proposed tool has the ability to offer new ways of investigating the intra and interindividual variations of the chickens based on the locomotion measurement. To the best of author's knowledge and based on web of science search, thigmotaxis of chickens in real-time with automated tracking has not been explored using experimental methods. Thigmotaxic responses of the chickens in responses to a stimulus or multiple stimuli or thermotaxis due to change in temperature conditions across the rearing floor of poultry industries can now be investigated using the proposed ChickTrack model. The recognition of early stage thigmotaxis will help to assess the chicken's spatial learning, cognitive performance, and memory. In the near future, new animal welfare indices can possibly be developed by relying only on the non-invasive way of automatic data collection and monitoring platforms for chickens. Tracking and the path trajectory measurement of chickens can be used as a proxy for anxiety based on the tendency of the birds to stay at the edges of the pen rather than staying in the center which is considered as bold. Further by using location and density to understand the dynamics of individual chicken birds, how they follow each other, exploring whether is there little to no movement for some individual birds then maybe that indicates illness or injury. Thigmotaxis are typically used as a measure of anxiety but not fear. The proposed tool can take advantage of the tracking module to determine thigmotaxis and thus anxiety of chickens can be estimated leading to an overall welfare estimate. Through digital phenotyping, correlations of these movement-based variations, the proposed model and associated automated monitoring sensor enabled technologies can aid in the productivity and welfare of poultry farming. Future research is warranted to investigate the proposed ChickTrack model and its contribution in the development of other animal welfare platforms. 


\section{Conclusions}

The height installation of the camera in the poultry barn, multiple viewpoints and angles in capturing the bird images will lead to specific characteristics of the chicken and its movement with varying scales, resolution and occlusions. The heterogeneous distribution in individual size and flock density causes challenges in the detection, ability to count and track movement of the chickens. There are currently no standards available, or any specific existing algorithms optimized for the movement tracking of chickens. The proposed Yolov5 model consists of this CSP backbone network, and the object detection function refines the flock density features via the deployment of the convolutional networks in the facilitation of the generation of trajectory movements, counting and tracking. The recall and the precision values of the ChickTrack model confirms the superior performance in the detection of the chickens in congested scenes, among various occlusions, and distribution density. Based on several experiments and video-based data analysis, the results showed that the proposed model for chicken detection, counting and tracking is robust and has the potential to be implemented for farm applications. The result of this study enhances the capacity to monitor both the individual and flock of chickens through enabling digitization and automated data processing in the poultry industry. The developed Yolov5 model has the potential to be used for other animals such as pigs, goats or cattle.

\section{Supplementary material}

Supplementary material namely sample videos S1 to S6 and the YoloV5 model are available for download.

\section{Declaration of competing interest}

The author being the sole contributor declare that there is no known competing financial interests or personal relationships that could have appeared to influence the work reported in this article.

\section{References}

1. Poultry global market report 2021: COVID-19 impact and recovery to 2030, Available at: https://www.researchandmarkets.com/reports/5240275/poultry-global-market-report2021-covid-19, Accessed on 18 July, 2021.

2. A. Willits-Smith, R. Aranda, M.C. Heller, et al., Addressing the carbon footprint, healthfulness, and costs of self-selected diets in the USA: a population-based crosssectional study, Lancet Planet. Health. 4(3) (2020) e98-e106. https://doi.org/10.1016/S2542-5196(20)30055-3.

3. S. Neethirajan, Automated tracking systems for the assessment of farmed poultry, Preprints (2021) 2021050364. http://dx.doi.org/10.20944/preprints202105.0364.v1.

4. A. Crump, G. Arnott, E.J. Bethell, Affect-Driven attention biases as animal welfare indicators: review and methods, Animals $8 \quad$ (2018) 136. https://doi.org/10.3390/ani8080136.

5. Global farm video surveillance system market size, status, and forecast 2021-2027, Available at: https://www.360researchreports.com/global-farm-video-surveillancesystem-market-17716906, Accessed on 18 July, 2021. 
6. S. Neethirajan, B. Kemp, Digital Livestock Farming, Sensing and Bio-Sensing Res. 32 (2021a) p.100408. https://doi.org/10.1016/j.sbsr.2021.100408.

7. S. Neethirajan, B. Kemp, Digital phenotyping in livestock farming, Animals 11 (2021b) 2009. https://doi.org/10.3390/ani11072009. requirements and feed utilization in ruminants, J. Agric. Sci. 155(7) (2017) 1188-1189. http://dx.doi.org/10.1017/S0021859617000296.

9. A. Mottet, G. Tempio, Global poultry production: current state and future outlook and challenges, Worlds Poult. Sci. J. 73(2) (2017) 245-256. https://doi.org/10.1017/S0043933917000071

10. S.N. Ismail, E.A. Awad, I. Zulkifli, et al., Effects of method and duration of restraint on stress hormones and meat quality in broiler chickens with different body weights, Asianaustralas J. Anim. Sci. 32(6) (2019) 865. https://doi.org/10.5713/ajas.18.0354.

11. L. O. Tedeschi, M. A. Fonseca, J. P. Muir, et al., A glimpse of the future in animal nutrition science. 2. Current and future solutions, Rev. Bras. Zootec. 46(5) (2017) 452-469. http://dx.doi.org/10.1590/s1806-92902017000500012.

12. L. A. González, I. Kyriazakis, L. O. Tedeschi, Review: Precision nutrition of ruminants: approaches, challenges and potential gains, Animal 12(s2) (2018) s246-s261. https://doi.org/10.1017/s1751731118002288.

13. N. Li, Z. Ren, D. Li, et al., Automated techniques for monitoring the behaviour and welfare of broilers and laying hens: towards the goal of precision livestock farming, Animal 14(3) (2020) 617-625. https://doi.org/10.1017/S1751731119002155.

14. S. Neethirajan, Transforming the adaptation physiology of farm animals through sensors, Animals 10(9) (2020) 1512. https://doi.org/10.3390/ani10091512.

15. L. O. Tedeschi, P. L. Greenwood, I. Halachmi, Advancements in sensor technology and decision support intelligent tools to assist smart livestock farming, J. Animal Sci. 99(2) (2021) 1-11. https://doi.org/10.1093/jas/skab038.

16. X. Yang, Y. Zhao, G.M. Street, et al., Classification of broiler behaviours using triaxial accelerometer and machine learning, Animal 15(7) (2021) 100269. https://doi.org/10.1016/j.animal.2021.100269.

17. A. Abdoli, S. Alaee, S. Imani, et al., Fitbit for chickens? time series data mining can increase the productivity of poultry farms, In Proceedings of the 26th ACM SIGKDD International Conference on Knowledge Discovery \& Data Mining (2020) 3328-3336. http://dx.doi.org/10.1145/3394486.3403385.

18. M. van der Sluis, Y. de Haas, B. de Klerk, et al., Assessing the activity of individual grouphoused broilers throughout life using a passive radio frequency identification system - a validation study, Sensors 20(13) (2020) 3612. http://dx.doi.org/10.3390/s20133612.

19. J.E. Del Valle, D.F. Pereira, M.M. Neto, et al., Unrest index for estimating thermal comfort of poultry birds (Gallus gallus domesticus) using computer vision techniques, Biosyst. Eng. 206, (2021) 123-134. https://doi.org/10.1016/j.biosystemseng.2021.03.018

20. A. Aydin, Using 3D vision camera system to automatically assess the level of inactivity in broiler chickens, Comput. Electron. Agric. 135 (2017) 4-10. https://doi.org/10.1016/j.compag.2017.01.024.

21. I. Nyalala, C. Okinda, C. Kunjie, et al., Weight and volume estimation of poultry and products based on computer vision systems: a review, Poult. Sci. (2021) 101072. https://doi.org/10.1016/j.psj.2021.101072. 
22. K. Wurtz, I. Camerlink, R.B. D'Eath, et al., (2019) Recording behaviour of indoor-housed farm animals automatically using machine vision technology: A systematic review. PLoS ONE 14(12) (2019) e0226669. https://doi.org/10.1371/journal.pone.0226669.

23. K. Cuan, T. Zhang, J. Huang, et al., Detection of avian influenza-infected chickens based on a chicken sound convolutional neural network, Comput. Electron Agric. 178 (2020) 105688. http://dx.doi.org/10.1016/j.compag.2020.105688.

24. H. Pu, J. Lian, M. Fan, Automatic recognition of flock behavior of chickens with convolutional neural network and kinect sensor, Intern. J. Pattern Recognit. Artif. Intell. 32(07) (2018) 1850023. https://doi.org/10.1142/S0218001418500234.

25. C.W. Ye, Z.W. Yu, R. Kang, et al., An experimental study of stunned state detection for broiler chickens using an improved convolution neural network algorithm, Comput. Electron Agric. 170 (2020) 105284. http://dx.doi.org/10.1016/j.compag.2020.105284.

26. C. Fang, T. Zhang, H. Zheng, et al., Pose estimation and behavior classification of broiler chickens based on deep neural networks, Comput. Electron Agric. 180 (2021) 105863. http://dx.doi.org/10.1016/j.compag.2020.105863.

27. L.D. Quach, N. Pham-Quoc, D.C. Tran, et al., Identification of chicken diseases using VGGNet and ResNet models, In International Conference on Industrial Networks and Intelligent Systems (2020) 259-269. http://dx.doi.org/10.1007/978-3-030-63083-6_20.

28. X. Zhuang, T. Zhang, Detection of sick broilers by digital image processing and deep $\begin{array}{lllll}\text { learning. } & \text { Biosyst. } & \text { Eng. } & 179 & \text { (2019) }\end{array}$ http://dx.doi.org/10.1016/j.biosystemseng.2019.01.003.

29. C.Y. Lin, K.W. Hsieh, Y.C. Tsai, et al., Monitoring chicken heat stress using deep convolutional neural networks, In 2018 ASABE Annual International Meeting (2018) 1. https://doi.org/10.13031/AIM.201800314.

30. V. Okinda, M. Lu, L. Liu, et al., A machine vision system for early detection and prediction of sick birds: A broiler chicken model, Biosyst. Eng. 188 (2019) 229-42. https://doi.org/10.1016/j.biosystemseng.2019.09.015.

31. D. Cheng, T. Rong, G. Cao, Density map estimation for crowded chicken, In International Conference on Image and Graphics (2019) 432-441. http://dx.doi.org/10.1007/978-3-03034113-8_36.

32. J. Wang, N. Wang, L. Li, et al., Real-time behavior detection and judgment of egg breeders based on YOLO v3. Neural. Comput. Appl. 32(10) (2020) 5471-5481. https://doi.org/10.1007/s00521-019-04645-4.

33. S. Tan, G. Lu, Z. Jiang, et al., Improved YOLOv5 network model and application in safety helmet detection, In 2021 IEEE International Conference on Intelligence and Safety for Robotics (ISR) (2021) 330-333. http://dx.doi.org/10.1109/ISR50024.2021.9419561.

34. Image polygonal annotation with python, Available at: http://labelme.csail.mit.edu/Release3.0, Accessed on 18 July, 2021.

35. K. Liu, H. Tang, S. He, et al., Performance validation of Yolo variants for object detection. In Proceedings of the 2021 International Conference on Bioinformatics and Intelligent Computing (2021) 239-243. http://dx.doi.org/10.1145/3448748.3448786.

36. G. Yang, W. Feng, J. Jin, et al., Face Mask Recognition System with YOLOV5 based on image recognition. In 2020 IEEE 6th International Conference on Computer and Communications (ICCC) (2020) 1398-1404. https://doi.org/10.1109/ICCC51575.2020.9345042. 
37. Y. Chen, C. Zhang, T. Qiao, et al., Ship detection in optical sensing images based on YOLOv5, In Twelfth International Conference on Graphics and Image Processing (ICGIP 2020) 11720 (2021) 117200E. http://dx.doi.org/10.3390/rs13050871.

38. F. Zhou, H. Zhao, Z. Nie, Safety helmet detection based on YOLOv5. In 2021 IEEE International Conference on Power Electronics, Computer Applications (ICPECA) (2021) 6-11. https://doi.org/10.1109/ICPECA51329.2021.9362711.

39. M. Kasper-Eulaers, N. Hahn, S. Berger, et al., Short communication: detecting heavy goods vehicles in rest areas in winter conditions using YOLOv5, Algorithms 14(4) (2021) 114. http://dx.doi.org/10.3390/a14040114.

40. Y. Fang, X. Guo, K. Chen, et al., Accurate and automated detection of surface knots on sawn timbers using YOLO-V5 model, BioResources 16(3) (2021) 5390-5406.

41. A. Kuznetsova, T. Maleva, V. Soloviev, YOLOv5 versus YOLOv3 for apple detection, Cyber-Physical Systems: Modelling and Intelligent Control (2021) 349-358. http://dx.doi.org/10.1007/978-3-030-66077-2_28.

42. J. Cho, Y. Jung, D. Kim, S., et al., Moving object detection based on optical flow estimation and a Gaussian mixture model for advanced driver assistance systems, Sensors 19(14) (2019) 3217. http://dx.doi.org/10.3390/s19143217.

43. N. Urieva, J. McDonald, T. Uryeva, et al., Collision detection and avoidance using optical flow for multicopter UAVs, In 2020 International Conference on Unmanned Aircraft Systems (ICUAS) (2020) 607-614. http://dx.doi.org/10.1109/ICUAS48674.2020.9213957
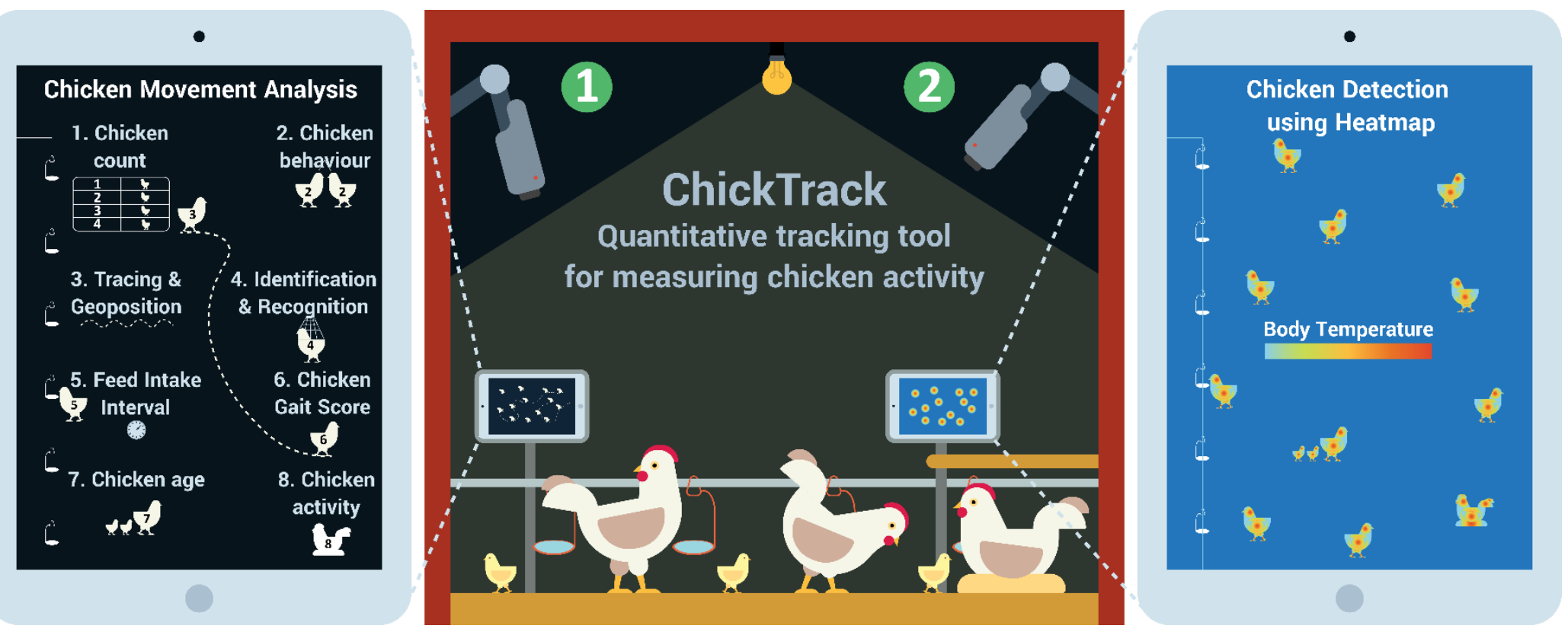